TITLE:

\title{
Exciton dephasing and multiexciton recombinations in a single carbon nanotube
}

\section{$\operatorname{AUTHOR}(\mathrm{S})$ :}

Matsuda, Kazunari; Inoue, Tadashi; Murakami, Yoichi; Maruyama, Shigeo; Kanemitsu, Yoshihiko

\section{CITATION:}

Matsuda, Kazunari ...[et al]. Exciton dephasing and multiexciton

recombinations in a single carbon nanotube. Physical Review B 2008, 77(3): 033406.

\section{ISSUE DATE:}

2008-01

URL:

http://hdl.handle.net/2433/87354

RIGHT:

c 2008 The American Physical Society 
PHYSICAL REVIEW B 77, 033406 (2008)

\title{
Exciton dephasing and multiexciton recombinations in a single carbon nanotube
}

\author{
Kazunari Matsuda, ${ }^{1, *}$ Tadashi Inoue, ${ }^{1}$ Yoichi Murakami, ${ }^{2}$ Shigeo Maruyama, ${ }^{2}$ and Yoshihiko Kanemitsu ${ }^{1,3, \dagger}$ \\ ${ }^{1}$ Institute for Chemical Research, Kyoto University, Uji, Kyoto 611-0011, Japan \\ ${ }^{2}$ Department of Mechanical Engineering, The University of Tokyo, 7-3-1 Hongo, Bunkyo, Tokyo 113-8656, Japan \\ ${ }^{3}$ Photonic and Electronic Science and Engineering Center, Kyoto University, Kyoto 615-8510, Japan
}

(Received 31 October 2007; published 8 January 2008)

\begin{abstract}
We studied the temperature and excitation intensity dependence of exciton luminescence in individual single-walled carbon nanotubes (SWNTs) using single nanotube photoluminescence (PL) spectroscopy. The linear temperature dependence of the PL linewidth in a single SWNT implies that the exciton dephasing is dominated by the interaction between the exciton and the phonon mode with very low energy under lowerexcitation conditions. Saturation of the PL intensity and broadening of the PL linewidth in a single SWNT occur simultaneously with an increase in excitation laser intensity. Our findings show that the rapid excitonexciton annihilation through multiparticle Auger recombination broadens the homogeneous PL linewidth.
\end{abstract}

DOI: 10.1103/PhysRevB.77.033406

PACS number(s): 78.67.Ch, 78.55.-m

A single-walled carbon nanotube (SWNT) of about $1 \mathrm{~nm}$ diameter and length greater than several hundreds of nanometers is a prototypical system of one-dimensional (1D) structures. $^{1}$ The electronic and optical properties of SWNTs recently have attracted a great deal of attention ${ }^{2}$ because the Coulomb interaction in the $1 \mathrm{D}$ system is strongly enhanced by the spatial carrier confinement. ${ }^{3}$ These enhanced Coulomb interactions lead to the formation of tightly bound excitons. Following theoretical studies of the SWNTs, ${ }^{4-6}$ the experimental studies support the existence of stable excitons in SWNTs. ${ }^{7,8}$ The characteristic optical properties of SWNTs are determined by the dynamics of 1D excitons with extremely large binding energies in the range of 200-400 meV.7,8 It was also expected that the strong Coulomb interaction would enhance the many body effects of excitons in a SWNT. Indeed, the recent experimental results of pump-probe and transient photoluminescence (PL) spectroscopy have revealed the existence of strong excitonexciton interactions under intense laser excitation. ${ }^{9-11}$

Exciton dephasing time and dephasing mechanisms play an important role in determining the optical properties of semiconductors and their device applications. In fact, the exciton dephasing containing fruitful physics has been extensively studied in low-dimensional semiconductors ${ }^{12,13}$ because this information will be necessary toward realization of quantum optical devices. However, the detailed exciton dephasing mechanisms in 1D SWNTs are not clear. The spectral linewidth of a single SWNT contains essential information on exciton dephasing time; therefore, PL measurements on single SWNTs ${ }^{14-18}$ must be performed to clarify the intrinsic spectral linewidth hidden by the inhomogeneously broadened ensemble-averaged PL spectra. In addition, the signatures of the exciton-exciton interaction and exciton dynamics appear in single SWNT PL spectra and their linewidths. The single carbon nanotube PL spectroscopy will provide new insight into the exciton dephasing and excitonexciton interactions in a SWNT, which cannot be obtained from ensemble-averaged PL and pump-probe spectroscopy.

In this Brief Report, we examine the temperature and excitation power dependence of PL spectra from spatially isolated single SWNTs using singe carbon-nanotube PL spectroscopy. The narrow Lorentzian PL spectrum is clearly observed, and the homogeneous PL linewidth linearly decreases with decreasing temperature. This linear temperature dependence implies that the exciton dephasing is dominated by the interaction between the exciton and the phonon mode with very low energy. In the high-excitation regime, the homogeneous linewidth broadens nonlinearly with an increase in excitation intensity. Our observation suggests that the broadening of homogeneous linewidth arises from the annihilation of excitons through a rapid Auger recombination process. The exciton-exciton interaction details concerning biexciton stability will be discussed.

The samples used in this study were spatially isolated SWNTs synthesized on Si substrates using an alcohol catalytic chemical vapor deposition method. ${ }^{19}$ The Si substrates were patterned with parallel grooves, typically $\sim 1 \mu \mathrm{m}$ wide and $500 \mathrm{~nm}$ deep using an electron-beam lithography technique. The isolated SWNTs grow from one side toward the opposite side of the groove. Several kinds of samples were prepared by changing the growth temperature and time. We used the SWNT sample without matrix and surfactant around the nanotubes to reduce the local environmental fluctuation effect. ${ }^{20}$ Single nanotube PL spectroscopy was performed on a sample grown at $750{ }^{\circ} \mathrm{C}$ for $30 \mathrm{~s}$ with a typical average luminescent SWNT number density of $0.1-1 / \mu \mathrm{m}^{2}$.

Single SWNT PL measurements were carried out at temperatures ranging from 4.7 to $90 \mathrm{~K}$ using a home-built confocal microscope. ${ }^{18}$ The SWNT samples were mounted in a cryostat and excited with either a He-Ne laser $(1.96 \mathrm{eV})$ or a mode-locked $\mathrm{Ti}: \mathrm{Al}_{2} \mathrm{O}_{3}$ laser $(1.72 \mathrm{eV})$. The pulse width and repetition rate of the $\mathrm{Ti}: \mathrm{Al}_{2} \mathrm{O}_{3}$ laser were $150 \mathrm{fs}$ and $80 \mathrm{MHz}$, respectively. The laser light was focused on the sample surface through a microscope objective. The PL signal from SWNTs was detected by a $30 \mathrm{~cm}$ spectrometer equipped with a liquid-nitrogen $\left(\mathrm{N}_{2}\right)$-cooled InGaAs photodiode array with a spectral range of $0.78-1.38 \mathrm{eV}$. The spectral resolution of the system was about $1.4 \mathrm{meV}$. The detector accumulation time was typically between 3 and $60 \mathrm{~s}$.

Figure 1(a) shows the temperature dependence of the PL spectra of an isolated SWNT excited with a He-Ne laser. The spectra were measured under lower-excitation conditions from 4.7 to $90 \mathrm{~K}$. The SWNT has a chiral index of $(10,3)$ 
(a)

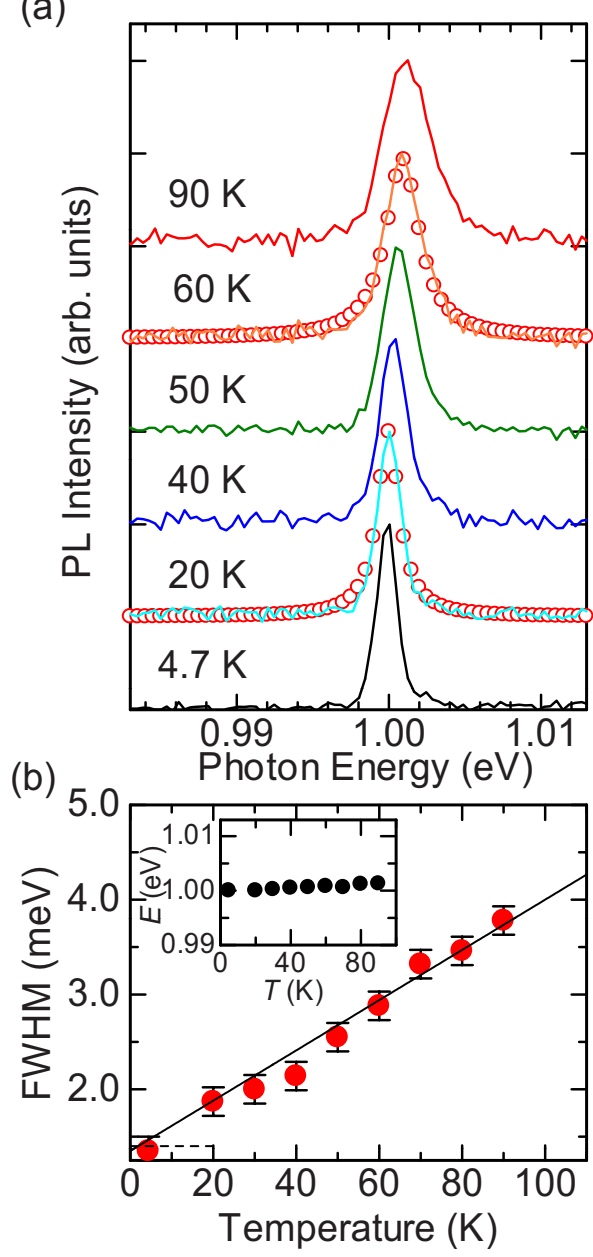

FIG. 1. (Color online) (a) Temperature dependence of PL spectra of a SWNT from 4.7 to $90 \mathrm{~K}$ excited with He-Ne laser light. Open circles denote the Lorentzian function fits. (b) Spectral linewidths [full width at half maximum (FWHM)] as a function of temperature. The dotted line represents the spectral resolution. The solid line indicates linear temperature dependence. The inset shows the temperature dependence of the PL peak energy.

based on the emission energies found in Refs. 21 and 22. The PL spectra clearly narrow when the temperature decreases. ${ }^{23}$ As indicated by the circles in the figure, the spectral line shapes are well fitted by Lorentzian functions. The linewidth [full width at half maximum (FWHM)] of a single SWNT, corresponding to a homogeneous linewidth, is plotted as a function of temperature in Fig. 1(b). The temperature dependence of the linewidth shows almost linear behavior (as indicated by a solid line) in the range of $5-90 \mathrm{~K}$. The inset of Fig. 1(b) shows the temperature dependence of the PL peak energy. The energy change from 5 to $90 \mathrm{~K}(\sim 1 \mathrm{meV})$ is much smaller than that in other compound semiconductor quantum wire (12 meV from 5 to $90 \mathrm{~K}$ in GaAs wires) in the weak exciton-phonon coupling regime. ${ }^{24}$ This weak temperature dependence is consistent with the experimental fact of narrow PL spectra with Lorentzian line shape in Fig. 1(a). (a)

(b)
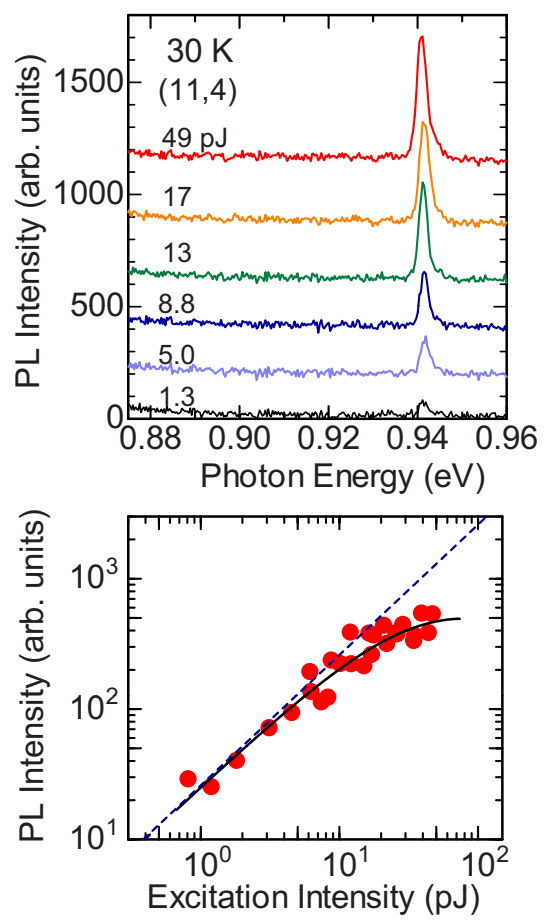

FIG. 2. (Color online) (a) Low-temperature PL spectra obtained from a typical single SWNT [assigned chiral index: $(11,4)$ ] at various excitation intensities for $1.72 \mathrm{eV}$ and $150 \mathrm{fs}$ pulses. (b) Integrated PL intensity as a function of the excitation laser intensity. As indicated by the dotted line, under weak excitation conditions, the PL intensities show almost linear power dependence. The solid line corresponds to the fitted curve.

Both the dephasing time and the lifetime of the excitons contribute to homogeneous linewidth broadening. The measured PL lifetimes of excitons are considerably longer (>20 ps) from 10 to $300 \mathrm{~K},{ }^{20}$ and this contribution to the linewidth is negligibly small $(\leqslant 0.1 \mathrm{meV})$ under lowerexcitation conditions. Thus, the temperature-dependent linewidths are determined approximately by the dephasing time of the exciton due to the exciton-phonon interactions. This linear temperature dependence implies that the very low energy phonon modes $\left(\ll k_{B} T\right)$ dominate the exciton dephasing. The contributed low energy phonon to the exciton dephasing is assigned as the TW (twisting) mode in SWNTs. ${ }^{18,25}$ Based on the homogeneous linewidth, the exciton dephasing time is evaluated from $350 \mathrm{fs}$ at $90 \mathrm{~K}$ to longer than $940 \mathrm{fs}$ at $5 \mathrm{~K}^{26}$

Figure 2(a) shows PL spectra obtained from a typical single SWNT [assigned chiral index: $(11,4)$ ] at $30 \mathrm{~K}$ and at various excitation intensities of $1.72 \mathrm{eV}$ and $150 \mathrm{fs}$ laser pulses. Each spectrum has a single peak located at $0.941 \mathrm{eV}$. Even in the high-excitation region above about $15 \mathrm{pJ}$ per pulse, the PL spectrum shows a single peak, without a change in the peak energy. Furthermore, additional spectral structures are not observed in any lower energy regions. Similarly, the PL bands due to biexcitons (M line) and inelastic exciton scattering (P line) $)^{3}$ are not observed in this spectral region.

Spectrally integrated PL intensities are plotted as a function of excitation laser intensity in Fig. 2(b). In a low- 
(a)

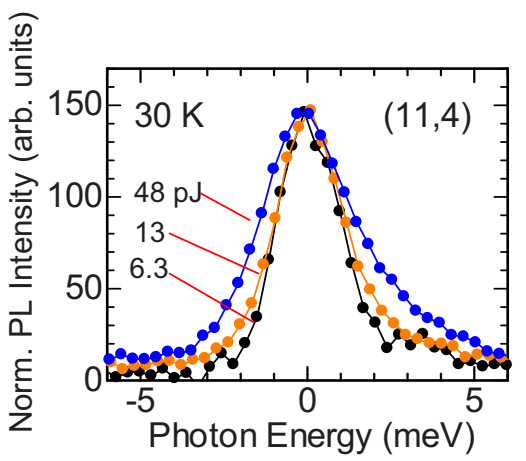

(b)

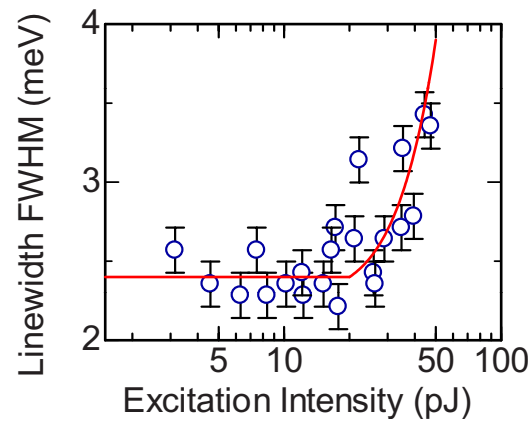

FIG. 3. (Color online) (a) Normalized PL spectra of a single SWNT on an expanded energy scale excited with different intensities. (b) Homogeneous linewidths as a function of excitation intensity. The solid line corresponds to the fitted curves considering exciton annihilation through the exciton-exciton scattering process.

excitation region below $10 \mathrm{pJ}$, the PL intensity grows almost linearly with excitation intensity (as indicated by a dotted line). Conversely, in the higher-excitation intensity region $(>20 \mathrm{pJ})$, saturation of the PL intensity is clearly apparent.

We show normalized PL spectra for a single SWNT, excited with different intensities, on an expanded energy scale in Fig. 3(a). The spectral linewidth broadens with increasing excitation intensity. The homogeneous linewidth is plotted in Fig. 3(b) as a function of excitation intensity, with the FWHM of the linewidth broadening nonlinearly. This broadening cannot be explained by laser heating effects because the nonlinear broadening behavior contradicts the linear temperature dependence of the linewidth broadening [see Fig. 1(b)]. This excitation intensity-dependent linewidth broadening indicates that the exciton dynamics are strongly affected by the multiple excitons present in a SWNT.

Under higher-excitation conditions, when additional homogeneous linewidth broadening and saturation of the PL intensity occur, more than one exciton is created in a single SWNT, leading to a remarkable scattering process between excitons. Exciton-exciton annihilation occurs due to the rapid Auger nonradiative recombination process through strong Coulomb interactions. ${ }^{27-29}$ The exciton annihilation rate $\left(1 / \tau_{\text {ex-ex }}\right)$ is given by ${ }^{10}$

$$
1 / \tau_{\mathrm{ex}-\mathrm{ex}}=A \operatorname{Ln}(n-1),
$$

where $A$ is the exciton annihilation (Auger) constant, $L$ $(\sim 1 \mu \mathrm{m})$ the length of the SWNT, and $n$ the time-averaged exciton number. The exciton number should be a discrete value. However, the exciton number fluctuates at each single excitation event. Then, we might consider that the timeaveraged exciton number $n$ changes continuously in the analysis of the time-averaged PL data. Taking the radiative lifetime ( $\tau_{\text {rad }}>1 \mathrm{~ns}$ ) (Ref. 30) and the nonradiative lifetime, due to the exciton trapping of the nonradiative centers $\left(\tau_{\text {nonrad }} \sim 30\right.$ ps) (Ref. 20$)$ into consideration, the PL intensity $I_{\mathrm{PL}}$ at the excitation power $P$ is described by

$$
I_{\mathrm{PL}} \propto \tau_{\text {rad }}^{-1} P /\left(\tau_{\text {rad }}^{-1}+\tau_{\text {nonrad }}^{-1}+\tau_{\text {ex }- \text { ex }}^{-1}\right) .
$$

The PL intensity saturation at higher-excitation regions, as shown in Fig. 2, can be explained by the opening of the nonradiative relaxation path due to the exciton-exciton annihilation.

The homogeneous linewidth $\Gamma$ in Fig. 3(b) is determined by both the excitation power independent exciton-phonon interaction term $\Gamma_{\text {ex-ph }}$ and the excitation power dependent Auger term $\Gamma_{\text {ex-ex }}\left(\propto 1 / \tau_{\text {ex-ex }}\right)$,

$$
\Gamma=\Gamma_{\text {ex-ph }}+\Gamma_{\text {ex-ex }} .
$$

The homogeneous linewidth broadening at higher-excitation regions, as shown in Fig. 3, can be explained by the shortening of exciton lifetime due to the Auger process. The nonlinear behaviors of the PL intensity and the homogeneous linewidth broadening can be reproduced using Eqs. (1)-(3) as indicated by the solid lines in Figs. 2(b) and 3(b). The time-averaged exciton number $n$ can be translated from the excitation intensity $P$ at which the PL intensity saturates. The best fitted curves in Figs. 2(b) and 3(b) with varying the fitting parameter $A$ reproduced the experimental results well. Then, the derived exciton-exciton annihilation constant $A$ of $1.6 \mathrm{ps}^{-1} \mu \mathrm{m}$ is almost consistent with the theoretically calculated value based on perturbation theory. ${ }^{10}$ From this result, it was found that the multiparticle Auger process occurs very efficiently with an Auger recombination time estimated at $800 \mathrm{fs}$ for $\sim 1-\mu \mathrm{m}$-long SWNT when two excitons are present in a SWNT. This very short time constant, in comparison to bulk semiconductors, is characteristics of lowdimensional systems, including SWNTs and is comparable to the exciton dephasing time at low temperature. These results indicate that the exciton dephasing is limited under highexcitation conditions, not by the exciton-phonon interactions but by the exciton-exciton interactions.

Under the highest-excitation conditions in this study, shown in Fig. 2(a), no spectral structures arising from exciton complexes, such as a biexciton, were observed in a lower energy region of the exciton PL line. However, Pedersen et $a l .{ }^{31}$ have discussed the exciton-exciton interaction and the stability of the biexciton in a SWNT based on the effectivemass theory. The biexciton binding energy is estimated at $37 \mathrm{meV}$ in a semiconducting SWNT with a $1.07 \mathrm{~nm}$ diameter [corresponding chirality: $(11,4)]$. This theoretical calculation predicts that the two excitons energetically form the bound biexciton when they are present in the SWNT. However, PL signals related to the biexciton emission were not experimentally observed, even under high-excitation and low-temperature conditions. In other pump-probe spectroscopy experiments, no transient absorption signal related to 
the biexciton has been observed. ${ }^{11}$ This inconsistency between theoretical prediction and the experimental results is attributable to the dynamic behavior of the excitons. As discussed above, the nonradiative Auger recombination process occurs efficiently at about $1 \mathrm{ps}$ order. As a result, one of the two excitons nonradiatively relaxes in the ground state when two excitons are present in a SWNT. The exciton-exciton scattering rate in a SWNT is much higher than in other 1D semiconductors, such as GaAs quantum wire, in which biexciton emission has been observed experimentally. ${ }^{32}$ The biexciton formation from two uncorrelated excitons and the exciton-exciton scattering process are compensated in a SWNT. Thus, the biexciton formation in a SWNT is obstructed because of the very efficient Auger recombination process resulting from the strong Coulomb interaction in 1D systems.

In conclusion, we performed a single carbon nanotube PL spectroscopy on single isolated SWNTs by changing the temperature and the excitation intensity. The homogeneous line- widths decreased linearly with a decrease in temperature. The temperature dependence implies that the exciton dephasing time was dominated by the interaction between the exciton and the phonon mode with very low energy. In the highexcitation regime, the homogeneous linewidth broadened nonlinearly with an increase in excitation intensity. Our observation suggests that the exciton-exciton annihilation through the rapid Auger recombination process contributes to the homogeneous broadening in a SWNT. It is pointed out that the instability of a biexciton in a carbon nanotube is due to the efficient exciton annihilation process.

The authors would like to thank S. Kasai and T. Ono for technical support in fabricating the grooved $\mathrm{Si}$ substrates for SWNT growth. Part of the work conducted at Kyoto University was supported by a Grant-in-Aid for Scientific Research from JSPS (KAKENHI Nos. 17684012 and 18340089), the Foundation for C\&C Promotion, and the Research Foundation for Opto-Science and Technology. *matsuda@scl.kyoto-u.ac.jp

†kanemitu@scl.kyoto-u.ac.jp

${ }^{1}$ S. Iijima, Nature (London) 354, 56 (1991).

${ }^{2}$ R. Saito, G. Dresselhaus, and M. S. Dresselhaus, Physical Properties of Carbon Nanotubes (Imperial, London, 1998).

${ }^{3}$ T. Ogawa, in Optical Properties of Low-Dimensional Materials, edited by T. Ogawa and Y. Kanemitsu (World Scientific, Singapore, 1995), p. 1.

${ }^{4}$ T. Ando, J. Phys. Soc. Jpn. 66, 1066 (1997).

${ }^{5}$ C. D. Spataru, S. Ismail-Beigi, L. X. Benedict, and S. G. Louie, Phys. Rev. Lett. 92, 077402 (2004).

${ }^{6}$ V. Perebeinos, J. Tersoff, and P. Avouris, Phys. Rev. Lett. 92, 257402 (2004).

${ }^{7}$ F. Wang, G. Dukovic, L. E. Brus, and T. F. Heinz, Science 308, 838 (2005).

${ }^{8}$ J. Maultzsch, R. Pomraenke, S. Reich, E. Chang, D. Prezzi, A. Ruini, E. Molinari, M. S. Strano, C. Thomsen, and C. Lienau, Phys. Rev. B 72, 241402(R) (2005).

${ }^{9}$ Y.-Z. Ma, L. Valkunas, S. M. Bachilo, and G. R. Fleming, J. Phys. Chem. B 109, 15671 (2005).

${ }^{10}$ F. Wang, Y. Wu, M. S. Hybertsen, and T. F. Heinz, Phys. Rev. B 73, 245424 (2006).

${ }^{11}$ G. N. Ostojic, S. Zaric, J. Kono, V. C. Moore, R. H. Hauge, and R. E. Smalley, Phys. Rev. Lett. 94, 097401 (2005).

${ }^{12}$ D. Gammon, E. S. Snow, B. V. Shanabrook, D. S. Katzer, and D. Park, Science 273, 87 (1996).

${ }^{13}$ W. Braun, M. Bayer, A. Forchel, H. Zull, J. P. Reithmaier, A. I. Filin, and T. L. Reinecke, Phys. Rev. B 56, 12096 (1997).

${ }^{14}$ A. Hartschuh, H. N. Pedrosa, L. Novotny, and T. D. Krauss, Science 301, 1354 (2003).

${ }^{15}$ J. Lefebvre, J. M. Fraser, P. Finnie, and Y. Homma, Phys. Rev. B 69, 075403 (2004).

${ }^{16}$ H. Htoon, M. J. O'Connell, P. J. Cox, S. K. Doorn, and V. I. Klimov, Phys. Rev. Lett. 93, 027401 (2004).
${ }^{17}$ K. Matsuda, Y. Kanemitsu, K. Irie, T. Saiki, T. Someya, Y. Miyauchi, and S. Maruyama, Appl. Phys. Lett. 86, 123116 (2005).

${ }^{18}$ T. Inoue, K. Matsuda, Y. Murakami, S. Maruyama, and Y. Kanemitsu, Phys. Rev. B 73, 233401 (2006).

${ }^{19}$ S. Maruyama, R. Kojima, Y. Miyauchi, S. Chiashi, and M. Kohno, Chem. Phys. Lett. 360, 229 (2002).

${ }^{20}$ H. Hirori, K. Matsuda, Y. Miyauchi, S. Maruyama, and Y. Kanemitsu, Phys. Rev. Lett. 97, 257401 (2006).

${ }^{21}$ S. M. Bachilo, M. S. Strano, C. Kittrell, R. H. Hauge, R. E. Smalley, and R. B. Weisman, Science 298, 2361 (2002).

${ }^{22}$ J. Lefebvre, J. M. Fraser, Y. Homma, and P. Finnie, Appl. Phys. A: Mater. Sci. Process. 78, 1107 (2004).

${ }^{23}$ J. Lefebvre, P. Finnie, and Y. Homma, Phys. Rev. B 70, 045419 (2004).

${ }^{24}$ A. V. Gopal, R. Kumar, A. S. Vengurlekar, T. Mélin, F. Laruelle, and B. Etienne, Appl. Phys. Lett. 74, 2474 (1999).

${ }^{25}$ J. Jiang, R. Saito, A. Gruneis, S. G. Chou, G. G. Samsonidze, A. Jorio, G. Dresselhaus, and M. S. Dresselhaus, Phys. Rev. B 71, 045417 (2005).

${ }^{26}$ The absolute value of the homogeneous linewidth depending on the diameter (chirality) changes from a nanotube to a nanotube (see Ref. 18).

${ }^{27}$ F. Wang, G. Dukovic, E. Knoesel, L. E. Brus, and T. F. Heinz, Phys. Rev. B 70, 241403(R) (2004).

${ }^{28}$ Y.-Z. Ma, L. Valkunas, S. L. Dexheimer, S. M. Bachilo, and G. R. Fleming, Phys. Rev. Lett. 94, 157402 (2005).

${ }^{29}$ L. Huang and T. D. Krauss, Phys. Rev. Lett. 96, 057407 (2006).

${ }^{30}$ V. Perebeinos, J. Tersoff, and P. Avouris, Nano Lett. 5, 2495 (2005).

${ }^{31}$ T. G. Pedersen, K. Pedersen, H. D. Cornean, and P. Duclos, Nano Lett. 5, 291 (2005).

${ }^{32}$ M. Yoshita, Y. Hayamizu, H. Akiyama, L. N. Pfeiffer, and K. W. West, Phys. Rev. B 74, 165332 (2006). 